\title{
Dimensões laborais, éticas e políticas do dimensionamento de pessoal de enfermagem diante da COVID-19
}

\author{
Labour, ethical and political dimensions of nursing staff sizing in the face of COVID-19 \\ Dimensiones laborales, éticas y políticas del dimensionamiento del \\ personal de enfermería frente a la COVID-19
}

Juliana Aparecida Peixoto Nishiyama ${ }^{1}$ (C) Rúbia Marcela Rodrigues Moraes $^{2}$ (c) Ana Maria Müller de Magalhães ${ }^{3}$ (i) Anair Lazzari Nicola ${ }^{4}$ (1) Denilse Damasceno Trevilato ${ }^{5,6}$ (1) João Lucas Campos de Oliveira ${ }^{3}$ (1)

1. Universidade Federal de Mato Grosso, Faculdade de Enfermagem, Programa de Pós-Graduação em Enfermagem. Cuiabá, MT, Brasil.

2. Hospital Universitário Júlio Müller, Programa de Pós-Graduação em Ciências Aplicadas à Atenção Hospitalar. Cuiabá, MT, Brasil.

3. Universidade Federal do Rio Grande do Sul, Escola de Enfermagem. Porto Alegre, RS, Brasil.

4. Universidade Estadual do Oeste do Paraná, Programa de Residência em Gerenciamento de Enfermagem em Clínica Médica e Cirúrgica. Cascavel, PR, Brasil.

5. Universidade Federal do Rio Grande do Sul, Escola de Enfermagem, Programa de Pós-Graduação em Enfermagem. Porto Alegre, RS, Brasil.

6. Hospital Moinhos de Vento. Porto Alegre, RS, Brasil.

Autor correspondente:

Juliana Aparecida Peixoto Nishiyama. E-mail: julynishiyama@gmail.com.

Recebido em 09/09/2020.

Aprovado em 10/11/2020

DOl:https://doi.org/10.1590/2177-9465-EAN-2020-0382

\section{Resumo}

Objetivo: propor discussão ampliada a respeito de dimensões que envolvem o dimensionamento de pessoal de enfermagem, articulando-as à realidade da pandemia por COVID-19. Método: ensaio teórico-reflexivo subsidiado por material técnico-científico e alusões acerca das dimensões/repercussões laborais, éticas e políticas do (sub)dimensionamento de pessoal de enfermagem e a realidade que o contexto da pandemia salientou na dinâmica de gestão de pessoas da categoria. Resultados: a reflexão fo conduzida por dois eixos: Dimensões do dimensionamento de pessoal de enfermagem e o cenário agravado pela COVID-19; e, Provimento de pessoal de enfermagem pós COVID-19: há otimismo? Considerações Finais e implicações para a prática: a situação sanitária expressa pela COVID-19, no Brasil, parece evidenciar para a sociedade a elevada carga de trabalho e a inadequação quantiqualitativa de profissionais de enfermagem. Isso reforçou a ambivalência de fortalezas e fragilidades das dimensões que envolvem os meios de previsão e provisão de recursos humanos. Numa proposição otimista, acredita-se que a articulação dos interesses da profissão, entidades de classe, órgãos governamentais, academia, gestores/instituições, além da sociedade como um todo, configura-se como um meio político de desdobrar o dimensionamento de profissionais de enfermagem e repercutir ética e positivamente nas condições laborais da categoria, além de favorecer a qualidade do cuidado.

Palavras-chave: Dimensionamento de pessoal; Carga de trabalho; Infecções por coronavirus; Equipe de enfermagem; Administração de recursos humanos.

\section{Abstract}

Aim: To propose a broader discussion on dimensions involving the sizing of nursing staff, articulating them to the reality of the pandemic by COVID-19. Method: Theoretical-reflexive essay subsidised by technical-scientific material and allusions about the labour, ethical and political dimensions/repercussions of the (sub)dimensioning of nursing staff and the reality that the context of the pandemic highlighted in the dynamics of management of people in the category. Results: the reflection was guided by two axes: Dimensions of nursing staff sizing and the scenario aggravated by COVID-19; and, Providing nursing staff after COVID-19: is there optimism? Final considerations and implications for practice: the health situation expressed by COVID-19, in Brazil, seems to evidence for society the high workload and the quantified inadequacy of nursing professionals This has reinforced the ambivalence of strengths and weaknesses of the dimensions involving the means of forecasting and the provision of human resources. In an optimistic proposition, it is believed that the articulation of the interests of the profession, class entities, government bodies, academia, managers/institutions, as well as society as a whole, is configured as a political means of unfolding the dimensioning of nursing professionals and having an ethical and positive impact on the working conditions of the category, as well as favouring the quality of care.

Keywords: Personnel downsizing; Workload; Coronavirus infections; Nursing, team; Personnel management.

\section{REsumen}

Objetivo: Proponer una discusión ampliada sobre las dimensiones que involucran el dimensionamiento del personal de enfermería, articulándolas a la realidad de la pandemia por COVID-19. Método: ensayo teórico-reflexivo subsidiado por material técnico-científico y alusiones acerca de las dimensiones/repercusiones laborales, éticas y políticas del (sub)dimensionamiento del personal de enfermería y la realidad que el contexto de la pandemia destacó, en la dinámica de gestión de personas de la categoría. Resultados: Dos ejes, condujeron la reflexión: Dimensiones del dimensionamiento del personal de enfermería y el escenario agravado por la COVID-19; y, Provisión del personal enfermería después de la COVID-19: ¿Hay optimismo? Consideraciones Finales e implicaciones para la práctica: La situación sanitaria expresada por la COVID-19, en Brasil, parece evidenciar para la sociedad, la elevada carga de trabajo y la inadecuación cuanticualitativa de los profesionales de enfermería. Esto reforzó la ambivalencia de fortalezas y las fragilidades de las dimensiones que involucran los medios previsión y provisión de los recursos humanos. Desde un punto de vista optimista, se cree que la articulación de los intereses de la profesión, entidades de clase, órganos gubernamentales, academia, gestores/instituciones e incluso de la sociedad como un todo, constituye un medio político para desdoblar el dimensionamiento de profesionales de enfermería y repercutir ética y positivamente en las condiciones laborales de la categoría, además de favorecer la calidad del cuidado.

Palabras clave: Reducción de personal; Carga de trabajo; Infecciones por coronavirus; Grupo de enfermería; Administración de personal. 


\section{INTRODUÇÃO}

Em dezembro de 2019, na cidade chinesa Wuhan, província de Hubei, registrou-se elevação de casos de síndrome respiratória aguda grave, cuja etiologia a princípio desconhecida foi posteriormente relacionada ao surgimento de um novo tipo de coronavírus, o SARS-CoV-2. ${ }^{1}$ A doença causada por esse agente etiológico foi definida como coronavirus disease-2019 (COVID-19) que, além da alta transmissibilidade, tem como características clínicas desde febre e tosse seca até insuficiência respiratória, falência de múltiplos órgãos e/ou choque séptico. ${ }^{2}$

Em 30 de janeiro de 2020, a Organização Mundial de Saúde (OMS) considerou a COVID-19 uma Emergência de Saúde Pública de Importância Internacional (ESPII), ${ }^{3}$ pois na mesma data a China registrava 7.734 casos e outros 90 casos foram relatados em países como Austrália, Canadá, Finlândia, França, Alemanha, Cingapura, República da Coréia, Emirados Árabes Unidos, Estados Unidos da América (EUA), Filipinas, Índia, Taiwan, Tailândia, Vietnã, Malásia, Nepal, Sri Lanka, Camboja e Japão. ${ }^{4}$ Em 11 de março de 2020, a OMS classificou a situação da COVID-19 como uma pandemia. ${ }^{3}$ Diante de tal realidade, líderes de governo, órgãos sanitários locais e internacionais em diferentes níveis elaboraram planos de contingência para tentar diminuir o impacto social e sanitário do contexto de potencial catastrófico. ${ }^{3,5}$

A OMS registrou até o dia 09 de novembro de 2020 , 50.266.033 casos confirmados da COVID-19, com 1.254 .567 óbitos. ${ }^{6}$ Até esta data, os EUA apresentavam a maior prevalência de casos confirmados (9.763.730) e de óbitos (235.562); a Índia ocupava a segunda posição em casos confirmados (8.553.657) e o Brasil a terceira, com 5.653.561 diagnósticos. Todavia, o Brasil é o segundo no ranking mundial em número de óbitos (162.269). ${ }^{6}$

Entre as estatísticas alarmantes da COVID-19, há registro de que 42.538 mil profissionais de enfermagem no Brasil contaminaram-se pelo novo coronavírus, com 460 óbitos, o que representa uma letalidade de 1,94\% entre os trabalhadores da área. ${ }^{7} \mathrm{O}$ maior volume de casos ocorreu nas regiões Sudeste (33,15\%) e Nordeste $(29,72 \%)$; o sexo feminino $(85,03 \%)$ foi o mais acometido, e a faixa etária predominante foi de 31 a 40 anos (17.825). ${ }^{7}$

Segund o o Conselho Federal de Enfermagem (COFEN) e o International Council of Nurses (ICN), o Brasil é o país com mais óbitos de profissionais de enfermagem pelo novo coronavírus no mundo. ${ }^{8}$ Essa fatal realidade se aguça com as condições de trabalho recorrentemente citadas como precárias para os trabalhadores de enfermagem brasileiros, o que se tornou ainda mais evidente no contexto de pandemia por COVID-19. ${ }^{9}$

De acordo com relatório da OMS e do ICN, existem cerca de 28 milhões de profissionais de enfermagem no mundo, ${ }^{10} \mathrm{e}$, no Brasil, são aproximadamente 2,2 milhões de trabalhadores que atuam em diferentes áreas e regiões. ${ }^{7}$ Apesar deste quantitativo se sobressair em uma comparação absoluta da enfermagem com outras categorias profissionais, estima-se que existe um déficit de quase 6 milhões de trabalhadores de enfermagem no mundo, com destaque para África, Sudeste Asiático e da região do Mediterrâneo Oriental (região da OMS), além de algumas partes da América Latina. ${ }^{11}$ Este cenário evidencia a necessidade de meios e instrumentos de previsão racional e satisfatória de pessoal de enfermagem, como o dimensionamento. ${ }^{12,13}$

Atualmente, no Brasil, os parâmetros utilizados para o dimensionamento do quadro de profissionais de enfermagem seguem a Resolução no 543/2017 do COFEN ${ }^{12}$ que, em tese, pode garantir a previsão adequada de pessoal em diferentes cenários de prática. No entanto, inadequações sobre esta ou mesmo a própria elevação da carga de trabalho da enfermagem são fatos verificados com frequência. ${ }^{13-15}$

A deficiência de força e condições de trabalho da enfermagem parece estar em voga no contexto de pandemia por COVID-19. Em todo o mundo seguem expressivos os índices de contaminação ${ }^{9}$ desta população, que é particularmente afetada por atuar no "front de batalha", e que também vivencia situações referentes ao aumento da carga de trabalho, privação de descanso, falta de equipamentos de proteção individual (EPI), constante medo de contaminar os familiares, desinformação da sociedade e descontentamento em relação às ações governamentais, dos sistemas de saúde e da população. ${ }^{16,17}$

Dado contínuo compromisso de suscitar debate que possa fundamentar incremento a respeito de melhores condições de trabalho da enfermagem, o que se torna inadiável no contexto de pandemia vigente e também para a posteridade esperada, este ensaio teórico-reflexivo tem o objetivo de propor discussão ampliada sobre as dimensões que envolvem o dimensionamento de pessoal de enfermagem, articulando-as à realidade da pandemia por COVID-19.

\section{MÉTODO}

Ensaio teórico-reflexivo, ancorado em material técnico-científico acerca da pandemia por COVID-19, e, principalmente, nas dimensões e repercussões na carga de trabalho e no (sub) dimensionamento de pessoal de enfermagem neste contexto emergente, no cenário prévio e agravado por ele, bem como de vislumbres futuros. Isso se deu em especial no contexto brasileiro, uma vez que existe organização do trabalho própria da profissão no País, além de meios de provimento e administração de recursos humanos de enfermagem identitários.

As alusões/inferências reflexivas propostas emanaram da junção da interpretação de dados científicos e material técnico do COFEN com a experiência autoral. Neste sentido, a reflexão foi sustentada por pesquisadores com experiência na área de gestão de pessoas em enfermagem e estudantes de pós-graduação em nível de mestrado e doutorado, sediados nos estados do Rio Grande do Sul, Mato Grosso e Paraná.

A fim de atender ao objetivo proposto de forma sistematizada o texto foi organizado e desenvolvido em dois eixos condutores, assim rotulados: Dimensões do dimensionamento de pessoal de enfermagem e o cenário agravado pela COVID-19; e, Provimento de profissionais de enfermagem pós COVID-19: há otimismo? 


\section{RESULTADOS E DISCUSSÃO}

\section{Dimensões do dimensionamento de pessoal de enfermagem e o cenário agravado pela COVID-19}

A enfermagem é imprescindível e reputada como essencial nos cuidados à saúde em todo o mundo. ${ }^{18}$ Corresponde à metade dos trabalhadores da saúde no Brasil e insere mais de 2 milhões de profissionais em nível superior (23\%) e médio (77\%) no cuidado à saúde no território nacional. Por isso, é impossível cogitar o desenvolvimento do trabalho das instituições de saúde sem sua a atuação. ${ }^{18}$ Por outro lado, o déficit quantiqualitativo de capital humano (subdimensionamento) - aqui entendido como uma das dimensões laborais do dimensionamento de pessoal de enfermagem -, é uma franca realidade que se perdura no tempo em muitos cenários de prática, ${ }^{19} \mathrm{e}$ algo em tela, até mesmo pela mídia, no contexto de pandemia hodierno.

Estudo ${ }^{20}$ realizado na Bélgica, Inglaterra, Finlândia, Irlanda, Espanha e Suíça, com 13.077 enfermeiros, constatou que o quantitativo inadequado de profissionais associado ao maior número de pacientes assistidos pode ocasionar sobrecarga de trabalho, insatisfação profissional e burnout. Aos pacientes, este desequilíbrio pode acarretar aumento de eventos adversos e maior taxa de mortalidade. ${ }^{21}$ Essa realidade delineia com clareza a necessidade de enfermeiros, gestores de enfermagem, entidades de classe e pesquisadores (re)planejarem continuamente meios de previsão e provisão de pessoal, como o dimensionamento.

O dimensionamento de pessoal de enfermagem é um método de previsão (planejamento) de profissionais da área, os quais, no Brasil, são devidamente projetados em número e categoria profissional para atender determinada demanda de clientela com qualidade e segurança, além de permear um meio de viabilização de melhores condições no trabalho. ${ }^{12,13,20}$ Apesar de sua relevância e possível eficácia como instrumento de gestão de pessoas, percebe-se a insuficiência de enfermeiros em todo o território nacional, principalmente no interior, já que 56,8\% residem e trabalham nas capitais, o que repercute negativamente na atuação deste profissional em prol da qualidade do cuidado, e fomenta a sobrecarga para os enfermeiros das localidades interioranas. ${ }^{19}$ Mesmo em cidades de médio ou grande porte e em regiões de alta densidade populacional, como a Sudeste e a Sul, são relatadas deficiências na adequação de profissionais de enfermagem (especialmente de nível superior), em diferentes níveis de complexidade assistencial. ${ }^{13-15}$

Com a pandemia de COVID-19, a enfermagem reafirma sua importância, tanto por ter o maior contingente de profissionais de saúde que está ininterruptamente prestando assistência ao paciente, como por possuir competência técnica especializada e uma visão holística sobre o cuidado. ${ }^{18}$ A OMS declarou que sem a atuação da enfermagem não será possível combater a pandemia, bem como será improvável alcançar os Objetivos de Desenvolvimento Sustentável (ODS) e a cobertura universal de saúde. ${ }^{11}$ Contudo, milhares de profissionais de saúde e de enfermagem, no Brasil e em diversos países como China e Itália, foram afastados das suas atividades laborais por terem adquirido COVID-19, e muitos morreram em decorrência do vírus, ${ }^{21,22}$ o que reforça que, sem a previsão e provisão adequada de trabalhadores de enfermagem, o enfrentamento da pandemia torna-se ainda mais desafiador (ou inviável).

O aumento da demanda de pacientes por trabalhador de enfermagem (logo, a elevação da carga de trabalho) compromete sobremaneira a qualidade do cuidado prestado e a segurança do paciente, estando associado estatisticamente à maior permanência hospitalar, maior infecção urinária relacionada a procedimentos invasivos e à menor satisfação de pacientes com o cuidado de enfermagem. ${ }^{23}$ Além disso, a sobrecarga do trabalho repercute em sensação de impotência, insatisfação e desmotivação nos profissionais. ${ }^{24}$

Em decorrência da redução do quantitativo dos trabalhadores na pandemia pelo absenteísmo-doença, além da sobrecarga de trabalho, profissionais de enfermagem expressam lesões por fricção e cisalhamento em consequência da utilização ininterrupta de EPI, em especial as máscaras N95/PFF2, óculos e a face shield, ${ }^{25}$ utilizados por jornadas de trabalho prolongadas que, ainda que necessárias para o enfrentamento da COVID-19, são um possível reflexo da escassez e/ou inadequação de profissionais prévia a este contexto.

A sobrecarga de trabalho torna-se ostensiva ante as demandas institucionais e a qualidade do cuidado prestado. Ademais, reconhece-se que não é atípico profissionais de enfermagem trabalharem mais de 60 horas semanais ${ }^{26} \mathrm{e} / \mathrm{ou}$ estarem sob sua responsabilidade um número excessivo de pacientes para prestação de cuidados. ${ }^{23,27}$ No cenário hodierno de intensas mudanças, isso talvez seja ainda mais aguçado, já que é comum que trabalhadores de enfermagem tenham de abdicar ou adiar alguns meios de qualidade de vida no trabalho (como o planejamento de férias), bem como se veem na necessidade de atender não só um volume acentuado de pacientes, como também uma clientela desconhecida e que demanda cuidados peculiares.

A baixa valorização e visibilidade da profissão de enfermagem é evidenciada pelas jornadas de trabalho extenuantes, escassez de insumos, arrocho de trabalhadores, condições de trabalho insalubres, sucateamento das instituições, além da baixa remuneração ${ }^{19,28,29}$ que influencia os profissionais a terem múltiplos vínculos empregatícios. Por consequência, favorece-se a ineficiência dos trabalhadores na implementação da assistência segura e de qualidade e o esgotamento físico e psíquico. ${ }^{30}$ Em razão desses e de outros fatores, na pandemia vigente e inclusive fora dela, os profissionais deixam de ausentar-se do trabalho (absenteísmo) para estarem presentes mesmo sob adoecimento ou limitação física e/ou psíquica (presenteísmo). ${ }^{31}$ Assim, depreende-se que a múltipla jornada de trabalho e as deficiências quantiqualitativas do pessoal de enfermagem foram intensificadas no contexto de pandemia por COVID-19, que impôs outras adversidades ao trabalho, logo, possivelmente propiciou o adoecimento dos profissionais.

A Organização Pan-Americana da Saúde (OPAS), com o intuito de atenuar os prejuízos dos profissionais de saúde 
ocasionados pela pandemia, recomendou condutas que compreendem a capacitação dos trabalhadores com o propósito de restringir a contaminação, provimento de EPI e de apoio psicossocial para diagnóstico de patologias, estresse e burnout, garantia de jornada de trabalho adequada, repouso, assim como pagamento das remunerações sem delonga, horas extras e licença médica. ${ }^{32}$ Por outro lado, e em especial no Brasil, verifica-se que os profissionais de enfermagem têm se deparado com escassez de EPI, insumos e capacitação, dimensionamento de recursos humanos inapropriado e baixa remuneração salarial; e, nesse sentido, pesquisadores aludem que é preciso esforço e mobilização por parte dos órgãos representativos da classe junto aos governantes para o estabelecimento de meios que possam garantir melhores condições laborais. ${ }^{33}$

Estudo realizado na China com profissionais de saúde que enfrentaram a pandemia de COVID-19 identificou problemas psicológicos, tais como depressão, ansiedade, insônia e angustia. ${ }^{34}$ Esses profissionais estão expostos a estressores adicionais como: o risco de se infectar e contaminar outros, em especial seus familiares, o aumento das responsabilidades laborais (sobrecarga de trabalho e atendimento a uma demanda desconhecida até então), além da redução do autocuidado em virtude da insuficiência de tempo e disposição. ${ }^{35}$ Deste modo, alude-se que, ainda que a pandemia traga uma série de responsabilidades e demandas adicionais ao usual, a deficiência na adequação de pessoal de enfermagem pode ser mais um fator que contribui negativamente para a resiliência dos trabalhadores que a enfrentam.

Perante o panorama da superlotação das unidades de internação, inclusive leitos de campanha, o COFEN ${ }^{36}$ estabeleceu parâmetros mínimos para o dimensionamento da equipe de enfermagem para a assistência aos pacientes infectados pela COVID-19 internados em unidades de terapia intensiva (UTI), semi-intensiva, hospitais gerais e de campanha. Como se trata de uma doença com um perfil clínico em construção, os pacientes em unidades de internação devem ser classificados inicialmente como de cuidados intermediários, requerendo seis horas de assistência de enfermagem/paciente/dia. Isto considerado, o cálculo resulta em um quadro de pessoal dimensionado composto por um percentual de $33 \%$ (mínimo de seis) de enfermeiros, nas 24 horas, a cada 20 leitos de internação e ajustado à carga horária semanal contratual da equipe. ${ }^{36}$

Considera-se que a estratégia do COFEN supracitada trata de uma dimensão ética (inerente à profissão enquanto classe) do dimensionamento de profissionais de enfermagem no contexto de pandemia; pois, ainda que de caráter emergencial e de base empírica (como o próprio COFEN ${ }^{36}$ assume), corresponde a uma resposta da entidade de classe a respeito de uma busca por prover parâmetros mínimos para a adequação do número e qualificação de trabalhadores esperados à demanda imposta pela COVID-19. Não menos importante, para além da qualificação em termos de categoria profissional determinada pelo dimensionamento, considera-se que a qualificação dos profissionais sob o prisma de capacitação é essencial para a disseminação do conhecimento, considerando que a educação permanente, articulada à implementação de protocolos e a práxis fundamentada em evidências, contribui para o aprimoramento da enfermagem. ${ }^{37}$

Mesmo com o disposto do COFEN, é comum que se vincule pela mídia, por exemplo, que no atual cenário da pandemia há escassez de profissionais capacitados e com experiência para o manejo adequado de equipamentos e cuidados complexos de enfermagem voltados a pacientes graves com COVID-19, além de um número insuficiente de trabalhadores já constatado diversas vezes em muitos cenários de prática antes da pandemia. ${ }^{13-15}$ Outro aspecto vinculado na mídia no período pandêmico foi a impossibilidade de abertura de alguns serviços emergenciais em razão da insuficiência e/ou inexistência de recursos humanos e outros necessários para a assistência. Destarte, este cenário contribui para o aumento da sobrecarga dos profissionais que estão neste "front", seja pela carga física e/ou emocional/ mental, tendo em vista que, por necessidade dos serviços, muitos profissionais tiveram de ser remanejados e passaram a desenvolver atividades complexas que não faziam parte de seu cotidiano. ${ }^{38}$

De acordo com o Parecer Normativo 02/2020 do COFEN, ${ }^{36}$ na assistência aos pacientes infectados pela COVID-19 e internados na UTI, a equipe deverá ser composta, no mínimo, por um enfermeiro a cada cinco leitos, um técnico de enfermagem a cada dois leitos e mais um técnico de enfermagem para apoio às atividades assistenciais a cada cinco leitos, em todos os turnos de trabalho. Já em unidades semi-intensivas, a proporção estipulada é de um enfermeiro para cada oito leitos, um técnico de enfermagem para cada dois leitos e mais um profissional de nível médio para apoio a cada oito leitos, em todo plantão/ turno de trabalho. ${ }^{36}$

O quantiqualitativo de trabalhadores de enfermagem deve ser assegurado para atendimento das necessidades dos pacientes, no entanto, o aumento da taxa de absenteísmo e presenteísmo durante a pandemia ${ }^{39}$ pode acarretar elevação de risco para o paciente e adoecimento da equipe de enfermagem pela excessiva carga de trabalho. Neste sentido, no contexto dos cuidados críticos, uma pesquisa italiana identificou que a carga de trabalho da equipe de enfermagem mensurada pelo Nursing Activities Score (NAS) foi significativamente maior quando no atendimento de pacientes com COVID-19. ${ }^{40}$

Outra particularidade que se desdobra no dimensionamento de profissionais de enfermagem na pandemia é o fato de que o COFEN estabeleceu um índice de segurança técnica (IST) de $20 \%$, em razão do expressivo aumento dos afastamentos desta categoria profissional, ${ }^{36}$ valor que difere do parâmetro mínimo estabelecido fora do contexto de pandemia, que é de $15 \% .{ }^{12}$ O IST tem a finalidade de atribuir um valor adicional ao quadro de pessoal de enfermagem para suprir as ausências previstas $e$ não previstas da equipe ${ }^{12}$ o que, no contexto de pandemia, por conta do excesso de trabalho e de afastamento/adoecimento de trabalhadores, esperam-se proporções aumentadas de ausências. Mais do que isso, é importante salientar que um estudo recente 
realizado antes do advento da COVID-19 no interior de São Paulo estimou um IST calculado em torno de $40 \%$ (42\% para enfermeiros e $38 \%$ para técnicos de enfermagem), ${ }^{41}$ ou seja, mesmo fora do contexto da pandemia, o absenteísmo é uma franca realidade da enfermagem e que pode ser relacionado à carga de trabalho e ao subdimensionamento de pessoal. ${ }^{42}$

Existe um déficit estimado de 23.961 profissionais de enfermagem no Brasil, sendo 8.430 enfermeiros e 15.531 técnicos/ auxiliares, ${ }^{41}$ o que prejudica a resposta adequada à pandemia de COVID-19 no País, e o atendimento fora dela. Durante o contexto pandêmico foram recebidas 8.680 denúncias pelos Conselhos Regionais de Enfermagem, as quais se referiam especialmente à escassez de $\mathrm{EPI}$, seguida pelo déficit de profissionais de enfermagem. ${ }^{43}$ Ademais, estudo identificou que o Brasil, a Espanha e Portugal têm dificuldades semelhantes em relação à escassez de enfermeiros e de recursos materiais, ${ }^{31}$ o que sinaliza que o problema de adequação quanti e/ou qualitativa de pessoal não é uma exclusividade brasileira.

Postula-se que existem dimensões políticas do dimensionamento em enfermagem no cenário de pandemia, bem como em contexto não pandêmico, uma vez que é perceptível que a adequação e o provimento da força de trabalho da categoria perpassam também por esferas decisórias que tendem a extrapolar os interesses diretos de profissionais e/ou lideranças de enfermagem. Ou seja, ainda que de forma correlata e até mesmo "velada", existem movimentos macro e micropolíticos que podem afetar a adequação do número e qualificação de trabalhadores de enfermagem disponíveis e atuantes em condições laborais dignas.

A Emenda Constitucional no 95, sancionada em 2016, ${ }^{44}$ que congelou o dispêndio público por 20 anos e impacta eminentemente o Sistema Único de Saúde (SUS) poderá interferir na provisão de profissionais de enfermagem, o que tensiona a sobrecarga de trabalho. No contexto da pandemia, é prudente ressaltar que o devido investimento no SUS é essencial para constatação do diagnóstico das infecções por coronavírus e sua restrição, entre outras doenças, ${ }^{45}$ o que inclui a adequada previsão e provisão do capital humano de enfermagem, já que atua incisiva e imprescindivelmente nestes combates.

A Medida Provisória (MP) 927/2020, ${ }^{46}$ por sua vez, flexibiliza as leis trabalhistas para o combate da pandemia, adapta as horas extras, a suspensão de exigências em segurança e saúde e altera o regulamento do trabalho: anteriormente, plantão realizado com 24 horas de trabalho previa 24 horas de descanso, já com a MP, outorga-se plantões de 24 horas com 12 horas de descanso. ${ }^{46}$ Neste sentido, ainda que seja pertinente refletir sobre medidas emergenciais de enfrentamento da pandemia, o que se propõe aqui é reafirmar, de fato, o quanto a enfermagem está exposta à precariedade em caso de não atendimento aos requisitos de adequação de profissionais, que pode ser viabilizada pelo dimensionamento. ${ }^{47} \mathrm{Em}$ outras palavras, o que parece é que, por um lado, medidas alteram a dinâmica laboral da profissão como uma forma de resposta à situação epidemiológica, e, por outro, ela se vê com uma falta prolongada de suporte estrutural, aqui enfocando àquele relacionado ao provimento de recursos humanos.

O Projeto de Lei (PL) no 2295/2000, que vem sendo debatido e analisado no Congresso Nacional, dispõe sobre a fixação da jornada de trabalho de 30 horas semanais e de seis horas diárias dos profissionais de enfermagem, ${ }^{48}$ sendo esta uma das principais reivindicações dos trabalhadores de enfermagem. Sabe-se que, em decorrência da excessiva carga de trabalho, da própria natureza do labor de enfermagem, bem como do estresse físico, psíquico e social, a luta pela redução da jornada de trabalho se posta como legítima para os trabalhadores da categoria. Contudo, cumpre ponderar que a obtenção das 30 horas semanais não deve reverberar na redução da remuneração dos profissionais, no arrocho de benefícios trabalhistas, ${ }^{49}$ tampouco no (maior) sucateamento qualiquantitativo do contingente de trabalhadores. Em claras palavras, um avanço não pode representar um ou mais retrocessos, e, articulando isso com a pandemia de COVID-19, espera-se que exista algum legado dela proveniente.

No ano internacional da enfermagem, também marcado pela pandemia de COVID-19, provoca-se a alteração da desfragmentação da assistência, propondo a partir da liderança dos enfermeiros a transformação ética e satisfatória para a saúde da sociedade. ${ }^{50} \mathrm{~A}$ campanha Nursing Now, lançada oficialmente no Brasil em 2019, prevê o fomento da liderança de enfermeiros e sua maior participação na agenda política e em posições decisórias. ${ }^{47}$ Espera-se que isso signifique um movimento de maior engajamento da profissão no que diz respeito ao poder de definir rumos, incluindo aspectos de interesse à adequação de profissionais de enfermagem, tanto no contexto de pandemia como fora dele, o que interpreta-se como plenamente ajustado à "Meta 2" da campanha, a qual trata do investimento em melhores condições de trabalho. ${ }^{51}$ Assim, emerge a necessidade de se refletir sobre o período pós-pandêmico.

\section{Provimento de profissionais de enfermagem pós COVID-19: há otimismo?}

A COVID-19 é uma patologia eminentemente contagiosa, com inexistência de vacinas e assistência terapêutica efetiva até o momento ${ }^{52}$ (outubro de 2020). O risco de colapso na saúde é existente, sendo imprescindível expor a vivência dos profissionais de saúde e de enfermagem durante o enfrentamento imposto. Considera-se fundamental que a sociedade condecore esses colaboradores imprescindíveis para que se sintam reconhecidos como fundamentais na assistência à saúde e no desenvolvimento do acesso e da qualidade dos serviços. ${ }^{53,54}$ Contudo, muito mais do que isso, os trabalhadores de enfermagem precisam que o reconhecimento social à sua bravura seja desdobrado em melhores condições de trabalho, o que inclui, com evidência, a adequação do provimento de pessoal.

Espera-se que os profissionais de enfermagem sejam reconhecidos financeira, social e profissionalmente, e que, em caso de legalização dos PL da carga horária de 30 horas semanais de trabalho e do piso salarial nacional, não haja abertura para retrocessos. Além disso, espera-se que pesquisas e 
reflexões inerentes às condições de trabalho e às consequências prejudiciais no processo do trabalho no decorrer da pandemia e no pós-pandemia sejam fomentadas com o intuito de elencar as intervenções efetivas para profilaxia e acompanhamento de agravos aos trabalhadores. ${ }^{33,53}$

Os órgãos governamentais devem ter como prioridade nas suas pautas a segurança e a saúde dos profissionais de saúde, assim como os gestores das instituições de saúde e dos serviços de enfermagem devem promover ambiente de trabalho seguro para os trabalhadores e pacientes. Ademais, as entidades da categoria (sindicatos, conselhos e associações de enfermagem) necessitam garantir as prerrogativas trabalhistas, melhores condições laborais e apoio psicossocial aos profissionais, seja no âmbito público ou privado. ${ }^{53}$

A pandemia da COVID-19 evidenciou a enfermagem no Brasil e no mundo. Além disso, vem comprovando a magnitude do SUS, dos centros de pesquisas e das universidades (ensino, pesquisa e extensão), os quais contribuem para uma sociedade mais empática aos grupos mais vulneráveis, ${ }^{54} \mathrm{e}$ são imprescindíveis para a viabilização de ações técnicas no enfrentamento da pandemia.

Sobre a previsão de possíveis cenários a respeito do provimento de profissionais de enfermagem no aclamado período pós-pandemia, não cabe afirmar de forma contundente quais serão e o quanto a pandemia impactará as decisões futuras. No entanto, cabe ponderar que a articulação de órgãos governamentais, fiscais, acadêmicos, de lideranças de enfermagem, dos próprios trabalhadores e da sociedade como um todo, pode ser um meio de valorizar de fato o trabalho da enfermagem e, assim, que as repercussões a respeito da adequação dos quadros de profissionais sejam mais factíveis e otimistas. Sabe-se que isso permeia um jogo de interesses por vezes distinto, e, para tanto, o processo de definição honesta e clara de metas que convirjam a um objetivo minimamente em comum pode ser interessante.

\section{CONSIDERAÇÕES FINAIS E IMPLICAÇÕES PARA A PRÁTICA}

A situação sanitária expressa pela COVID-19, no Brasil, parece evidenciar para a sociedade a elevada carga de trabalho e o subdimensionamento de profissionais de enfermagem. Este cenário também demonstra a ambivalência de fortalezas e fragilidades das dimensões que envolvem o dimensionamento de recursos humanos como instrumento de gestão de pessoas na categoria.

$\mathrm{Na}$ dimensão laboral, evidencia-se e são reforçadas as repercussões deletérias que a inadequação de pessoal de enfermagem acarreta aos trabalhadores, pacientes e serviços, em múltiplas esferas. Numa visão ética, pontua-se que as iniciativas de órgãos fiscais e regulatórios da profissão sobre a definição de parâmetros que determinem o dimensionamento de pessoal de enfermagem na pandemia e fora dela são profícuas, ainda que isso por si só não garanta a adequação esperada, até mesmo porque fica evidente que existe uma dimensão política que envolve o dimensionamento de recursos humanos. Neste prisma, pondera-se que os interesses (des)articulados macro e micropolíticos da profissão, entidades de classe, governo, serviços e lideranças de saúde/enfermagem, pesquisadores/ universidades, e a própria sociedade como um todo tendem a se firmar positiva ou negativamente no cenário de previsão e provisão de pessoal de enfermagem, seja no enfrentamento da pandemia (de forma austera) ou após isso.

Acredita-se que a limitação mais expressiva deste estudo é o fato de terem sido tecidas reflexões críticas à guisa de uma perspectiva sem, no entanto, terem sido consideradas outras possíveis, como as próprias dificuldades que gestores e instituições podem encontrar para melhorarem o provimento de profissionais de enfermagem, principalmente no contexto atribulado da pandemia por COVID-19. Todavia, pondera-se que o estudo tem um efeito teorizante robusto que pode servir de subsídio para debates que venham a suscitar melhores práticas de dimensionamento e adequação de pessoal de enfermagem e, desta forma, repercutir na qualidade do cuidado, em especial no contexto brasileiro que foi mais amplo e profundamente explorado.

\section{FINANCIAMENTO}

O presente trabalho foi realizado com apoio da Coordenação de Aperfeiçoamento de Pessoal de Nível Superior - Brasil (CAPES).

\section{CONTRIBUIÇÕES DOS AUTORES}

Desenho do estudo de reflexão. Juliana Aparecida Peixoto Nishiyama. Rúbia Marcela Rodrigues Moraes. Ana Maria Müller de Magalhães. Anair Lazzari Nicola. Denilse Damasceno Trevilato. João Lucas Campos de Oliveira.

Levantamento de informações. Juliana Aparecida Peixoto Nishiyama. Rúbia Marcela Rodrigues Moraes.

Reflexão analítica sobre as fontes téoricas. Juliana Aparecida Peixoto Nishiyama. Rúbia Marcela Rodrigues Moraes. Ana Maria Müller de Magalhães. Anair Lazzari Nicola. João Lucas Campos de Oliveira.

Interpretação dos resultados. Juliana Aparecida Peixoto Nishiyama. Rúbia Marcela Rodrigues Moraes. Ana Maria Müller de Magalhães. Anair Lazzari Nicola. Denilse Damasceno Trevilato. João Lucas Campos de Oliveira.

Redação e revisão crítica do manuscrito. Juliana Aparecida Peixoto Nishiyama. Rúbia Marcela Rodrigues Moraes. Ana Maria Müller de Magalhães. Anair Lazzari Nicola. Denilse Damasceno Trevilato. João Lucas Campos de Oliveira.

Aprovação da versão final do artigo. Juliana Aparecida Peixoto Nishiyama. Rúbia Marcela Rodrigues Moraes. Ana Maria Müller de Magalhães. Anair Lazzari Nicola. Denilse Damasceno Trevilato. João Lucas Campos de Oliveira.

Responsabilidade por todos os aspectos do conteúdo e a integridade do artigo publicado. Juliana Aparecida Peixoto Nishiyama. Rúbia Marcela Rodrigues Moraes. Ana Maria Müller de Magalhães. Anair Lazzari Nicola. Denilse Damasceno Trevilato. João Lucas Campos de Oliveira. 


\section{EDITOR ASSOCIADO}

\author{
Cristina Rosa Baixinho
}

\section{REFERÊNCIAS}

1. Li Q, Guan X, Wu P, Wang X, Zhou L, Tong Y et al. Early transmission dynamics in Wuhan, China, of Novel Coronavirus-Infected Pneumonia. N Engl J Med. 2020;382(13):1199-207. http://dx.doi.org/10.1056/ NEJMoa2001316. PMid:31995857.

2. Iser BPM, Sliva I, Raymundo VT, Poleto MB, Schuelter-Trevisol F, Bobinski F. Suspected COVID-19 case definition: a narrative review of the most frequent signs and symptoms among confirmed cases. Epidemiol Serv Saude. 2020 jun 22;29(3):e2020233. http://dx.doi. org/10.5123/S1679-49742020000300018. PMid:32609142.

3. World Health Organization, Organização Pan-americana da Saúde. Folha informativa-COVID-19 (doença causada pelo novo coronavírus) [Internet]. Genebra:WHO; 2020 [citado 2020 jun 30]. Disponível em: https://www. paho.org/bra/index.php?option=com_content\&view=article\&id=6101: covid 19\&ltemid=875

4. Bassetti M, Vena A, Giacobbe DR. The Novel Chinese Coronavirus (2019-nCoV) Infections: challenges for fighting the storm. Eur J Clin Invest. 2020;50(3):e13209. http://dx.doi.org/10.1111/eci.13209. PMid:32003000.

5. Ministério da Saúde (BR), Secretaria de Vigilância em Saúde. Novo coronavírus (2019-nCoV). Boletim Epidemiológico [Internet]; 2020 [citado 20 jun 2020]; 4(51):1-37. Disponível em: https://www.conasems. org.br/wp-content/uploads/2020/01/Boletim_epidemiologico_SVS_04. pdf

6. World Health Organization. Coronavirus Disease (COVID-19) Dashboard [Internet]. Genebra: WHO; 2020 [citado 2020 out 14]. Disponível em: https://covid19.who.int/

7. Conselho Federal de Enfermagem. Observatório da Enfermagem [Internet]. Brasília: COFEN; 2020 [citado 2020 out 14]. Disponível em: http://observatoriodaenfermagem.cofen.gov.br/

8. Conselho Federal de Enfermagem. Brasil é o país com mais mortes de enfermeiros por Covid-19 no mundo [Internet]. Brasília: COFEN 2020 [citado 2020 ago 1]. Disponível em: http://www.cofen.gov.br/ brasil-e-o-pais-com-mais-mortes-de-enfermeiros-por-covid-19-nomundo-dizem-entidades_80181.html

9. Fiho JMJ, Assunção AÁ, Algranti E, Garcia EG, Saito CA, Maeno M. A saúde do trabalhador e o enfrentamento da COVID-19. Rev Bras Saúde Ocup. 2020;45:e14. http://dx.doi.org/10.1590/2317-6369ed0000120.

10. Organização Pan-Americana de Saúde. Dia Mundial da Saúde: OMS e parceiros pedem investimentos urgentes em profissionais de enfermagem [Internet]. OPAS Brasil;2020 [citado 2020 ago 26]. Disponível em: https://www. paho.org/bra/index.php?option=com_content\&view=article\&id=6134:diamundial-da-saude-oms-e-parceiros-pedem-investimentos-urgentesem-profissionais-de-enfermagem\&ltemid $=844$

11. World Health Organization. State of the world's nursing 2020 [Internet] Genebra:WHO;2020 [citado 2020 jun 20]. Disponível em: https://apps. who.int/iris/bitstream/handle/10665/331673/9789240003293-eng.pdf

12. Resolução COFEN n ${ }^{\circ} 543,18$ de abril de 2017 (BR). Atualiza e estabelece parâmetros para o dimensionamento do quadro de profissionais de enfermagem nos serviços/locais em que são realizadas atividades de enfermagem. Diário Oficial da União [periódico na internet]. Brasília (DF), 2017 [citado 2020 jul 18]. Disponível em: http://www.cofen.gov. br/resolucao-cofen-5432017 51440.htm

13. Vasconcelos RO, Rigo DFH, Marques LGS, Nicola AL, Tonini NS, Oliveira JLC. Dimensioning of hospital nursing personnel: study with brazilian official parameters of 2004 and 2017. Esc Anna Nery. 2017;21(4):e20170098. http://dx.doi.org/10.1590/2177-9465-ean-2017-0098.

14. Santos LC, Andrade J, Spiri WC. Dimensioning of nursing professionals implications for the work process in the family health strategy. Esc Anna Nery. 2019;23(3):e20180348. http://dx.doi.org/10.1590/2177-9465ean-2018-0348.

15. Maziero ECS, Teixeira FFR, Cruz EDA, Matsuda LM, Sarquis LMM Dimensionamento de pessoal de enfermagem em unidades de terapia intensiva infantil: carga de trabalho versus legislação. Cogitare Enfermagem [Internet]. 2020; [citado 2020 jul 26];25:e64058. Disponível em: https://revistas.ufpr.br/cogitare/article/view/64058

16. Barbosa DJ, Gomes MP, Souza FBA, Gomes AMT. Fatores de estresse nos profissionais de enfermagem no combate à pandemia da COVID-19: Síntese de Evidências. Com. Ciênc Saúde (Porto Alegre) [Internet]. 2020; [citado 2020 jul 26];31(Suppl 1):31-47. Disponível em: http://www.escs. edu.br/revistaccs/index.php/comunicacaoemcienciasdasaude/article/ view/651/291

17. Helioterio MC, Lopes FQRS, Sousa CC, Souza FO, Pinho PS, Sousa FNF et al. Covid-19: por que a proteção de trabalhadores e trabalhadoras da saúde é prioritária no combate à pandemia? Trab Educ Saúde. 2020;18(3):e00289121. http://dx.doi.org/10.1590/1981-7746-sol00289.

18. Silva MCN, Machado MH. Sistema de Saúde e Trabalho: desafios para a Enfermagem no Brasil. Ciênc. saúde coletiva. 2020;25(1):7-13. http:// dx.doi.org/10.1590/1413-81232020251.27572019.

19. Fugulin FMT, Oliveira JLC, Nicola AL, Araujo ASS, Marinho AM, Canavezi CM et al. Dimensionamento de profissionais de enfermagem: implicações para a prática assistencia. Divulgação em Saúde para Debate [Internet]. 2016; [citado 2020 jul 30];56:126-33. Disponível em: https:// www.researchgate.net/publication/311753919_Dimensionamento_de profissionais_de_enfermagem_implicacoes_para_a_pratica_assistencia

20. Aiken LH, Sloane D, Griffiths P, Rafferty AM, Bruyneel L, McHugh M et al Nursing skill mix in European hospitals: cross-sectional study of the association with mortality, patient ratings, and quality of care. BMJ Qual Saf. 2017;26(7):559-68. http://dx.doi.org/10.1136/bmjqs-2016-005567. PMid:28626086.

21. Conselho Federal de Enfermagem. Saúde de Profissionais de Enfermagem é foco em tempos de Covid-19 [Internet]. Brasília: COFEN; 2020. [citado 2020 jun 20]. Disponível em: http://www.cofen.gov.br/saude-deprofissionais-de-enfermagem-e-foco-em-tempos-de-covid-19_78321. $\mathrm{html}$

22. The Lancet. COVID-19: protecting health-care workers. Lancet 2020;395(10228):922. http://dx.doi.org/10.1016/S0140-6736(20)30644-9.

23. Magalhães AMM, Costa DG, Riboldi CO, Mergen T, Barbosa AS, Moura GMSS. Association between workload of the nursing staff and patient safety outcomes. Rev Esc Enferm USP. 2017;51(0):e03255. http:// dx.doi.org/10.1590/s1980-220x2016021203255. PMid:29211232.

24. Biff D, Pires DEP, Forte ECN, Trindade LL, Machado RR, Amadigi FR et al. Cargas de trabalho de enfermeiros: luzes e sombras na Estratégia Saúde da Família. Ciênc. saúde coletiva. 2020;25(1):147-58. https:// doi.org/10.1590/1413-81232020251.28622019.

25. Ramalho AO, Freitas PSS, Nogueira PC. Medical Device-Related Pressure Injury in health care professionals in times of pandemic ESTIMA. Braz. J. Enterostomal Ther. 2020;18:e0120. http://dx.doi. org/10.30886/estima.v18.867_IN.

26. Santos RR, Lima EFA, Freitas PSS, Galavote HS, Rocha EMS, Lima RCD. The role of teamwork in Primary Health Care. Rev. Bras. Pesq. Saúde [Internet]. 2016; [citado 2020 jul 10];18(1):130-39. Disponível em: https:// pdfs.semanticscholar.org/8171/df67d2254cd879c258afd7b0254ae96a9311. pdf

27. Pires DEP, Machado RR, Soratto J, Scherer MA, Gonçalves ASR, Trindade LL. Nursing workloads in family health: implications for universal access. Rev. Latino-Am. Enfermagem. 2016;24(0):e2677. http://dx.doi. org/10.1590/1518-8345.0992.2682.

28. Melo CMM, Carvalho CA, Silva LA, Leal JAL, Santos TA, Santos HS Nurse workforce in state services with direct management: revealing precarization. Esc Anna Nery. 2016;20(3):e20160067. http://dx.doi. org/10.5935/1414-8145.20160067.

29. Oliveira AC. Challenges faced by nursing professionals in the COVID19 pandemic [editorial]. REME - Rev Min Enferm. 2020;24:e-1302. http:/ dx.doi.org/10.5935/1415-2762.20200032.

30. Falcão DA, Macedo AMA, Sousa VM, Fernandes KJSS, Pereira FGF Nursing team stress at the ready-service of a public hospital. Rev. Enferm. UFPI. 2019;8(2):38-44.

31. Mosteiro-Díaz MP, Baldonedo-Mosteiro M, Borges E, Baptista P, Queirós C, Sánchez-Zaballos M et al. Presenteeism in nurses: comparative study of Spanish, Portuguese and Brazilian nurses. Int Nurs Rev. 2020;00:110. PMid:32844446 
32. Organização Pan-Americana de Saúde. Surto da doença coronavírus (COVID-19): direitos, papéis e responsabilidades dos trabalhadores da saúde, incluindo as principais considerações sobre segurança e saúde ocupacional. Orientação provisória: 19 de março de 2020. OPAS/BRA/Covid-19/20-033 [Internet]. 2020 [citado 2020 jul 26]. Disponível em: https://iris.paho.org/bitstream/handle/10665.2/51988/ OPASBRACOVID1920033_por.pdf?sequence=1\&isAllowed=y

33. Quadros A, Fernandes MTC, Araujo BR, Caregnato RCA. Desafios da enfermagem brasileira no combate da COVID-19: uma reflexão. Enferm. Foco [Internet]. 2020; [citado 2020 ago 14];11(1 Esp):78-83. Disponível em: http://revista.cofen.gov.br/index.php/enfermagem/article/ view/3748/807

34. Lai J, Ma S, Wang Y, Cai Z, Hu J, Wei $\mathrm{N}$ et al. Factors associated with mental health outcomes among health care workers exposed to coronavirus disease 2019. JAMA Netw Open. 2020 mar;3(3):e203976. http://dx.doi.org/10.1001/jamanetworkopen.2020.3976. PMid:32202646.

35. Inter-Agency Standing Committee. Guia preliminar: como lidar com os aspectos psicossociais e de saúde mental referente ao surto de COVID-19-Versão 1.5 [Internet]. 2020 [citado 2020 jun 20]. Disponível em https://interagencystandingcommittee.org/system/files/2020-03/ IASC\%2OInterim\%20Briefing\%20Note\%20on\%20COVID-19\%20 Outbreak\%20Readiness \%20and\%20Response\%200perations\%20 -\%20MHPSS\%20\%28Portuguese\%29.pdf

36. Parecer Normativo $\mathrm{n}^{\circ}$ 002/2020 - atualização 01, de 28 de maio de 2020 (BR). Estabelece, na forma deste Parecer Normativo, parâmetros mínimos de profissionais de Enfermagem para atendimento aos pacientes acometidos pela COVID-19, internados em Hospitais Gerais, Hospitais de Campanha, Unidades de Tratamento Semi-Intensivo/ Salas de Estabilização e Unidades de Terapia Intensiva-UTI. Diário Oficial da União [periódico na internet]. Brasília (DF), 2020 [citado 2020 jun 20]. Disponível em: http://www.cofen.gov.br/parecer-normativono-002-2020_79941.html

37. Duncan DL. What the COVID-19 pandemic tells us about the need to develop resilience in the nursing workforce. Nurs Manag (Harrow). 2020;27(3):22-7. http://dx.doi.org/10.7748/nm.2020.e1933. PMid:32400142.

38. Ministério da Saúde (BR), Secretaria de Vigilância em Saúde. Doença pelo Coronavírus 2019 (COVID-19). Boletim Epidemiológico [Internet]; 2020 [citado 17 ago 2020]; 11:1-37. Disponível em: https://portalarquivos. saude.gov.br/images/pdf/2020/April/18/2020-04-17---BE11---Boletimdo-COE-21h.pdf

39. Maltezou HC, Dedoukou X, Tseroni M, Tsonou P, Raftopoulos V, Papadima $\mathrm{K}$ et al. SARS-CoV-2 infection in healthcare personnel with high-risk occupational exposure: evaluation of seven-day exclusion from work policy. Clin. infect dis. 2020;ciaa888. https://doi.org/10.1093/cid/ciaa888.

40. Lucchini A, Giani M, Elli S, Villa S, Rona R, Foti G. Nursing Activities Score is increased in COVID-19 patients. Intensive Crit Care Nurs. 2020;59:102876. http://dx.doi.org/10.1016/j.iccn.2020.102876. PMid:32360493.

41. Trettene AS, Razera APR, Beluci ML, Prado PC, Mondini CCSD, Spiri WC. Absenteeism and the Technical Safety Index of a tertiary hospital nursing team. Rev Esc Enferm USP. 2020;54:e03585. http://dx.doi. org/10.1590/s1980-220x2018036003585. PMid:32813797.

42. Feldhaus C, Souza RF, Fernandes LM, Carvalho ARS, Bordin V, Oliveira JLC. Association between workload and absenteeism in nursing technicians. Texto Contexto Enferm. 2019;28:e20180307. http://dx.doi. org/10.1590/1980-265x-tce-2018-0307.
43. Conselho Federal de Enfermagem. Fiscalização aponta subdimensionamento na resposta à COVID-19 [Internet]. Brasília: COFEN; 2020 [citado 2020 ago 17]. Disponível em: http://www.cofen.gov.br/fiscalizacao-apontasubdimensionamento-na-resposta-a-covid-19_81197.html

44. Emenda Constitucional no 95, de 15 de dezembro de 2016 (BR). Altera o Ato das Disposições Constitucionais Transitórias, para instituir o Novo Regime Fiscal, e dá outras providências. Diário Oficial da União [periódico na internet], Brasília (DF), 2016 [citado 17 ago 2020]. Disponível em: http://www.planalto.gov.br/ccivil_03/constituicao/emendas/emc/emc95. $\mathrm{htm}$

45. Rafael RMR, Neto M, Carvalho MMB, David HMSL, Acioli S, Faria MGA. Epidemiologia, políticas públicas e pandemia de Covid-19: o que esperar no Brasil?. Rev enferm UERJ. 2020;28:e49570. http://dx.doi. org/10.12957/reuerj.2020.49570.

46. Medida provisória nº 927, de 22 de março de 2020 (BR). Dispõe sobre as medidas trabalhistas para enfrentamento do estado de calamidade pública reconhecido pelo Decreto Legislativo ํㅡㄴ, de 20 de março de 2020, e da emergência de saúde pública de importância internaciona decorrente do coronavírus (covid-19), e dá outras providências. Diário Oficial da União [periódico na internet], Brasília (DF), 2020 [citado 30 jun 2020].Disponível em: http://www.planalto.gov.br/ccivil_03/_ato20192022/2020/Mpv/mpv927.htm

47. Silva LC, Oliveira DAL, Santos ABR, Barbosa LMS, Araújo LG, Barboza MTV et al. Dimensionamento de pessoal e sua interferência na qualidade do cuidado. Rev enferm UFPE on line. 2019;13(1):491-8. https://doi. org/10.5205/1981-8963-v13i02a236551p491-498-2019.

48. Projeto de Lei № 2295/2000 (BR). Dispõe sobre a jornada de trabalho dos Enfermeiros, Técnicos e Auxiliares de Enfermagem. Diário Oficial da União [periódico na internet], Brasília (DF), 2000 [citado 28 ju 2020]. Disponível em: https://www.camara.leg.br/proposicoesWeb/ fichadetramitacao?idProposicao $=17915$

49. Bardaquim VA, Dias EG, Dalri RCMB, Robazzi MCC. Reflexão sobre as condições de trabalho da enfermagem: subsídio às 30 horas de trabalho. Rev Enferm Contemp. 2019;8(2):171-81. http://dx.doi. org/10.17267/2317-3378rec.v8i2.2466.

50. Baixinho CL, Ferreira Ó. Defragment or integrate care? A challenge for the international year of the nurse. Rev baiana enferm. 2020:34:e.35856 http://dx.doi.org/10.18471/rbe.v34.35856.

51. Cassiani SHB, Lira No JCG. Nursing perspectives and the "Nursing Now" campaign. Rev Bras Enferm. 2018;71(5):2351-2. http://dx.doi. org/10.1590/0034-7167.2018710501. PMid:30304161.

52. Basile C, Combe C, Pizzarelli F, Covic A, Davenport A, Kanbay M et al Recommendations for the prevention, mitigation and containment of the emerging SARS-CoV-2 (COVID-19) pandemic in haemodialysis centres. Nephrol Dial Transplant. 2020;35(5):737-41. http://dx.doi. org/10.1093/ndt/gfaa069. PMid:32196116.

53. Machado MH, Pereira EJ, Ximenes No FRG, Wermelinger MCMW. Enfermagem em tempos da COVID-19 no Brasil: um olhar da gestão do trabalho. Enferm. Foco [Internet]; 2020; [citado 2020 ago 16];11(1 Esp):32-9. Disponível em: http://revista.cofen.gov.br/index.php/ enfermagem/article/view/3994/800

54. Geremia DS, Vendruscolo C, Celuppi IC, Souza JB, Schopf K, Maestri E. Pandemia covid-2019: formação e atuação da enfermagem para o Sistema Único de Saúde. Enferm. Foco [Internet]; 2020 [citado 2020 ago 16];11(1 Esp):40-7. Disponível em: http://revista.cofen.gov.br/index. php/enfermagem/article/view/3956 